\title{
Smad4 suppresses the progression of renal cell carcinoma via the activation of forkhead box protein $\mathrm{H1}$
}

\author{
YUNLI LIU ${ }^{1}$, YANGYANG XU ${ }^{2}$, XUEDONG LI ${ }^{1}$ and ZHAOYAN CHEN ${ }^{1}$ \\ ${ }^{1}$ Department of Urological Surgery, The Second Affiliated Hospital of Harbin Medical University; \\ ${ }^{2}$ Department of Urological Surgery, The First Affiliated Hospital of Harbin Medical University, \\ Harbin, Heilongjiang 150001, P.R. China
}

Received January 23, 2014; Accepted October 31, 2014

DOI: $10.3892 / \mathrm{mmr} .2014 .3061$

\begin{abstract}
Smad4 has recently been identified as a tumor suppressor gene in a variety of cancers, yet the role of Smad4 in renal cell carcinoma ( $\mathrm{RCC}$ ) remained to be elusive. Therefore, the aim of the present study was to explore the function of Smad4 in RCC. The expression of Smad4 reduced the growth rate of RCC. The levels of Smad4 and forkhead box protein H1 (FOXH1) mRNA were reduced, while the levels of estrogen receptor were increased in RCC cells compared with those in human renal epithelial cells $(\mathrm{P}<0.01)$. Western blot analysis showed an identical trend among the three molecules. Glutathione S-transferase pull-down and immunoprecipitation assays proved the interaction between Smad4 and FOXH1. An immunofluorescence assay revealed that Smad4 and FOXH1 were colocalized in the nuclei of RCC cells. Smad4 interacts with Smad2 and migrates into the nucleus, where it interacts with FOXH1 to repress the protein expression of estrogen receptor. These results indicate that $\mathrm{Smad} 4$ acts as a tumor suppressor by activating FOXH1, and then suppressing the expression of estrogen receptor, in addition to tumor migration and invasion. Hence, Smad4 should be investigated as a potential target for the treatment for RCC.
\end{abstract}

\section{Introduction}

Renal cell carcinoma ( $\mathrm{RCC}$ ) is one of the primary causes of cancer-associated mortalities, and its incidence is increasing $(1,2)$. Although there are numerous methods of detecting localized RCC, in the majority of cases the disease is difficult to diagnose (3). Furthermore, it is still controversial whether immunotherapy and antiangiogenic therapy are effective treatments for RCC $(4,5)$. Hence, it is necessary to find

Correspondence to: Dr Zhaoyan Chen, Department of Urological Surgery, The Second Affiliated Hospital of Harbin Medical University, 246 Xuefu Road, Harbin, Heilongjiang 150001, P.R. China

E-mail: zfchongqing@163.com

Key words: estrogen receptor, forkhead box protein H1, renal cell carcinoma, Smad4 novel biomarkers for the diagnosis and treatment of this disease. Additionally, the study of the pathways involved in the pathogenesis of RCC may offer further options for the treatment of RCC (6). Smad4 has been identified as a tumor suppressor gene in various cancer types (7-9). A higher frequency of Smad4 inactivation was observed in liver metastases than in extrahepatic metastases (10) and colorectal cancer patients expressing high Smad4 levels have been shown to have a longer survival time than the patients with low levels (11). Smad4 increases the levels of signaling in renal tubulointerstitial cells in a mouse model of renal disease (12). However, the potential role and molecular events of Smad4 signaling in RCC have remained elusive.

Smads are the central components of the intracellular signaling pathway of transforming growth factor- $\beta$ (TGF- $\beta$ ) ligands (13). The signaling molecules for activins include the two receptor-regulated Smads (R-Smads) Smad2 and Smad3, which are phosphorylated by ActRIB and form a heteromeric complex with Smad4 (13). The R-Smad/Smad4 complex translocates to the nucleus, where forkhead box protein H1 (FOXH1) interacts with the R-Smad-Smad4 complex to regulate transcription (14). The function of FOXH1 was initially identified as binding the Mix.2 gene in Xenopus. FOXH1 interacts with Smad4 and either phosphorylated Smad2 or Smad3 to form a complex (15). FOXH1 requires Smad to regulate transcription, as it does not contain a domain for the activation of transcription (16). FOXH1 inhibits the transcription of estrogen receptors (ERs) and androgen receptors (17). The FOXH1-R-Smad-Smad4 complex (CFSS) represses the transcriptional activities of ERs. Estrogen has been reported to induce renal carcinogenesis in Syrian hamsters (18). Thus, the CFSS may repress the development of RCC via the inhibition of the transcriptional activity of estrogen receptors. The aim of the present study was to investigate whether Smad4 is involved in the progression of RCC.

\section{Materials and methods}

Antibodies and reagents. Rabbit anti-Myc monoclonal antibody ( $1 \mathrm{mg} / \mathrm{ml}$; 1:500; used for the co-immunoprecipitation assay) was purchased from Clontech Laboratories (Mountain View, CA, USA). Mouse anti-Flag M2 monoclonal antibody (1 mg/ml; 1:2,000; used for fluorescence staining) was from Sigma-Aldrich (St. Louis, MO, USA). Mouse anti-green fluorescence protein (GFP) monoclonal antibody $(1 \mathrm{mg} / \mathrm{ml}$; 
1:1,000; used for fluorescence staining) was from Cell Signaling Technology Inc. (Danvers, MA, USA). Mouse anti-glutathione S-transferase monoclonal antibody (GST; $0.5 \mathrm{mg} / \mathrm{ml} ; 1: 1,000$; used for the assay of the results of GST-pulldown analysis). Mouse anti-GAPDH monoclonal antibody $(1 \mathrm{mg} / \mathrm{ml} ; 1: 1,000)$ and complementary horseradish peroxidase-labeled goat anti-mouse secondary antibodies $(1 \mathrm{mg} / \mathrm{ml} ; 1: 5,000)$ were purchased from Santa Cruz Biotechnology (Dallas, Texas, USA). Mouse anti-Smad4 (1:1,000), mouse anti-FOXH1 $(1: 1,000)$ and mouse anti-estrogen $(1: 1,000)$ monoclonal receptor antibodies (1 $\mathrm{mg} / \mathrm{ml}$ each, used for western blot analysis) were purchased from Shengshi Zhongfang BioSci \& Tech (Beijing, China). Mouse anti- $\beta$-actin monoclonal antibody $(1: 1,000$; loading control for western blot analysis) was purchased from Abcam (Shanghai, China). Phospho-Smad2 (Ser465/467) (138D4) rabbit monoclonal antibody $(1 \mathrm{mg} / \mathrm{ml} ; 1: 1,000$; \#3108; used for western blot analysis) was purchased from Cell Signaling Technology, Inc.

Renal cell cultures. The OS-RC-2 human RCC cell line was purchased from Riken Cell Bank (Tsukuba, Japan). The normal human renal cell line (HRE) was purchased from Promocell Co. Ltd. (Heidelberg, Germany). Cells were cultured in a humidified atmosphere of $5 \% \mathrm{CO}_{2}$ and $95 \%$ air at $37^{\circ} \mathrm{C}$ in RPMI-1640 medium (Gibco, Inc., Billing, MT, USA) supplemented with $10 \%$ heat-inactivated fetal calf serum (FCS; Shengma Yuanheng, Beijing, China). RCC and HRE proliferation was determined by direct counting. For direct counting, $10^{5}$ cells were seeded, harvested following three days of culture, and counted using a Hausser Scientific hemocytometer (Hausser Scientific, Horsham, PA, USA).

Plasmid constructs. Full-length Smad4 (forward, 5'-GAC ATCCATATGGACAATATGTCTATTAC-3' and reverse, 5'-GAC TGACTCGAGGTCTAAAGGTTGTGGGTC-3'), FOXH1 (forward, 5'-GACATCCATATGGGGCCCTGCAGCGGCTC-3' and reverse, 5'-GACTGACTCGAGCAGGCTGCACCAGGA GAG-3') and estrogen receptor molecules (forward, 5'-GACATC CATATGACCATGACCCTCCACAC-3' and reverse, 5'-GAC TGACTCGAGGACTGTGGCAGGGAAACCC-3'), FOXH1 and estrogen receptor molecules were constructed by polymerase chain reaction (PCR), followed by subcloning into various vectors at the sites of NdeI and XhoI (underlined). PCR was performed using EPPENDORF Mastercycler ${ }^{\circledR}$ nexus (Eppendorf AG, Hamburg Germany). Cycling conditions were as follows: $95^{\circ} \mathrm{C}$ for $1 \mathrm{~min}, 30$ cycles of $95^{\circ} \mathrm{C}$ for $20 \mathrm{sec}, 60^{\circ} \mathrm{C}$ for $30 \mathrm{sec}$ and $68^{\circ} \mathrm{C}$ for $2 \mathrm{~min}$ and one cycle of $68^{\circ} \mathrm{C}$ for $10 \mathrm{~min}$. The vectors were amplified in Escherichia (E.) coli, isolated using a QIAprep Miniprep kit (Qiagen Inc., Chatsworth, CA, USA) and verified using an ABI 3730 automatic DNA sequencer (Auke Biotech Co., Ltd, Beijing, China) with four dye fluorescence-based DNA sequencing.

Smad4expressionconstructs. TheSmad4 gene(accessionnumber, AB043547.1) was amplified using the following primers: Sense, 5'-GTGAGCTAGCATGGACAATATGTCTATTAC-3', and antisense, 5'-CTGAGAATTCCTTTATATATGCACTTGG-3', which generated a 1328 -bp product. The PCR product was cloned into the NheI-EcoRI sites of the pcDNA3.1 vector according to the manufacturer's instructions (TOPO TA
Expression kit; Invitrogen, Carlsbad, CA, USA), which was named as pcDNA3.1-Smad4. The pcDNA3.1-Smad4 plasmid was amplified in E. coli, isolated using a QIAprep Miniprep kit (Qiagen Inc.) and verified using an ABI 3730 automatic DNA sequencer with four dye fluorescence-based DNA sequencing.

RNA interference. The siRNA directed against Smad4 (5'-ATGTGCCATAGACAAGGTGGAG-3') and the non-target control siRNA (5'-UUCUCCGAACGUGUCACGU-3') were synthesized by Shanghai GenePharma (Shanghai, China).

Transfection of the RCC cell line. OS-RC-2 human RCC cells $\left(2 \times 10^{5}\right.$ per p-96 plate) were transfected with various vectors. Transfection was performed on cells in plates at $60 \%$ confluence using $9 \mu \mathrm{l}$ Lipofectamine 2000 ${ }^{\mathrm{TM}}$ (Applied Biosystems, Life Technologies, Foster City, CA, USA). Cells were split $48 \mathrm{~h}$ post-transfection and neomycin-resistant clones (G418; Sigma-Aldrich) were selected. Resistant colonies were either pooled or cloned by ring isolation.

Quantitative reverse transcription $P C R(R T-q P C R)$. Total RNA was isolated from the cells using QIAshredder and RNeasy Mini kits (Qiagen, Inc.). An initial strand of cDNA was synthesized from $500 \mathrm{ng}$ of RNA extracts in a volume of $20 \mu \mathrm{l}$ using avian myeloblastosis virus reverse transcriptase XL (Takara Biotechnology Co., Ltd., Dalian, China) priming with random 9 -mers at $42^{\circ} \mathrm{C}$ for $10 \mathrm{~min}$. The cDNA strand was stored at $20^{\circ} \mathrm{C}$ prior to use. Smad2, Smad4, FOXH1 and estrogen receptor transcriptional levels were estimated using RT-qPCR and qPCR. qPCR was conducted using SYBR Green I Master mix in a Light-Cycler 480, both obtained from Roche (Mannheim, Germany). RNA was isolated from the non-transfected and transfected OS-RC-2 cells, followed by cDNA synthesis and data analysis as described previously (7). The primers for $\mathrm{qPCR}$ were as follows: Sense, 5'-TACTATGTCTACTTCCTGAG-3', and antisense, 5'-CAAGGAAAATAAAACATACC-3' for Smad2; sense, 5'-ATTGATCTCTCAGGATTAAC-3', and antisense, 5'-GTGGTAGTGCTGTTATGATG-3' for Smad4; sense, 5'-ACTGAAGCTGGCCCAGATCA-3' and antisense, 5'-GGCCCAGGTCCTTGGCGAAG-3' for FOXH1; sense, 5'-TACCAATGACAAGGGAAGTA-3' and antisense, 5'-TGTTTCAACATTCTCCCTCC-3' for estrogen receptor and sense, 5'-CCCTTCATTGACCTCAACTAC-3', and antisense, 5'-CCACCTTCTTGATGTCATCAT-3' for GAPDH. GAPDH was used as an internal control. The AmpliTaq Gold enzyme (Applied Biosystems, Foster City, CA, USA) was activated by heating for $10 \mathrm{~min}$ at $95^{\circ} \mathrm{C}$, and all genes were amplified by 50 cycles of $15 \mathrm{sec}$ at $95{ }^{\circ} \mathrm{C}$, followed by $1 \mathrm{~min}$ at $60^{\circ} \mathrm{C}$.

To normalize for differences in the amount of total RNA added to each reaction mixture, GAPDH was used as an endogenous control. The data represent the average expression levels of the target genes, relative to GAPDH, from three independent cultures.

Renal cancer tissue collection. The study of human subjects and informed consent documents were approved by The Human Research Ethics Committee of The Second Affiliated Hospital of Harbin University (Harbin, China). From May 7th, 2011 to September 8th, 2012, a total of 102 RCC patients and 40 healthy 
subjects were recruited at The Second Affiliated Hospital of Harbin University. Potential confounding and mediating factors were identified in the associations between sedentary lifestyles and adiposity, which may be closely associated with the risk of RCC. During the survey, the body mass index (BMI) of participants was calculated using the formula: BMI $=$ weight $[\mathrm{kg}] /(\text { height }[\mathrm{m}])^{2}$.

The diagnostic requirements were included in the RCC group. The diagnostic criteria for RCC were used as in a previous study (19). There are four stages of RCC (stages I-IV). A number of tissue samples were obtained from The Second Affiliated Hospital of Harbin University with prior approval from the Institutional Review Board. Renal cancer or normal tissue biopsies were collected via a cystoscope and maintained below $-80^{\circ} \mathrm{C}$. A total of 142 tissue samples (normal tissue, $n=40$; RCC tissue, $\mathrm{n}=32$ for stage I, $\mathrm{n}=28$ for stage II, $\mathrm{n}=24$ for stage III and $n=18$ for stage IV) were distinguished by a pathologist experienced in renal cancer at the Department of Urology, Harbin Medical University.

Western blot analysis. RCC and HRE cells were homogenized in radioimmunoprecipitation assay buffer (Millipore, Billerica, MA, USA), consisting of $150 \mathrm{mM}$ sodium chloride, $1 \% \mathrm{NP}-40$, $0.5 \%$ sodium deoxycholate, $0.1 \%$ SDS, $50 \mathrm{mM}$ Tris- $\mathrm{HCl}(\mathrm{pH} 8.0)$ and cOmplete Mini Protease Inhibitor Cocktail (Roche). Once the debris was removed, the resulting supernatants were boiled and mixed with an equal volume of $20 \%$ glycerol containing $0.02 \%$ bromophenol blue (Beijing F\&F Chemical Industrial Co., Ltd., Beijing, China). Proteins were separated by 12\% SDS-PAGE (Beijing JingKeHongDa Biotechnology Co., Ltd) and transferred onto a polyvinylidene difluoride membrane (Millipore, Billerica, MA, USA). The membranes were blocked with 5\% skim med milk in Tris-buffered saline with Tween 20 [10 mM Tris (pH 7.5), $100 \mathrm{mM} \mathrm{NaCl}$ and $0.1 \%$ Tween 20; Dako, Carpinteria, CA, USA] and incubated with primary antibodies for Smad4, FOXH1, estrogen receptor, $\beta$-actin and phospho-Smad2 (Ser465/467) (138D4), in TBST with 0.5\% skimmed milk overnight at $4^{\circ} \mathrm{C}$. The membrane was treated with a peroxidase-conjugated secondary antibody $(1: 3,000)$ (GE Healthcare, Pittsburgh, PA, USA). Immunoreactive bands were visualized by enhanced chemiluminescence (RPN2132; GE Healthcare) in the chamber of the Chemiluminescence Analyzer system CLA-FS4 (Tohoku ${ }^{\mathrm{TM}}$ Electronic lnc., Miyagi, Japan), and quantified by densitometry with Image J software version 1.45 (National Institutes of Health, Bethesda, MD, USA).

GST pull-down assay. Bacteria-expressed GST or GST-Smad4 proteins were immobilized on glutathione-Sepharose 4B beads (GE Healthcare), washed, and the beads were incubated with FOXH1. The beads were washed with GST binding buffer $(100 \mathrm{mM} \mathrm{NaCl}, 50 \mathrm{mM} \mathrm{NaF}, 2 \mathrm{mM}$ EDTA and $1 \%$ Nonidet P40; Beijing JingKeHongDA Biotechnology Co., Ltd, Beijing, China) and proteins were eluted, followed by western blotting.

Immunoprecipitation. Cells were harvested and lysed in HEPES lysis buffer [20 mM HEPES (pH 7.2), $50 \mathrm{mM}$ $\mathrm{NaCl}, 0.5 \%$ Triton X-100, $1 \mathrm{mM} \mathrm{NaF}, 1 \mathrm{mM}$ dithiothreitol; Beijing JingKeHongDA Biotechnology Co., Ltd]. The lysate was incubated with the antibodies for Myc and GST for $3 \mathrm{~h}$

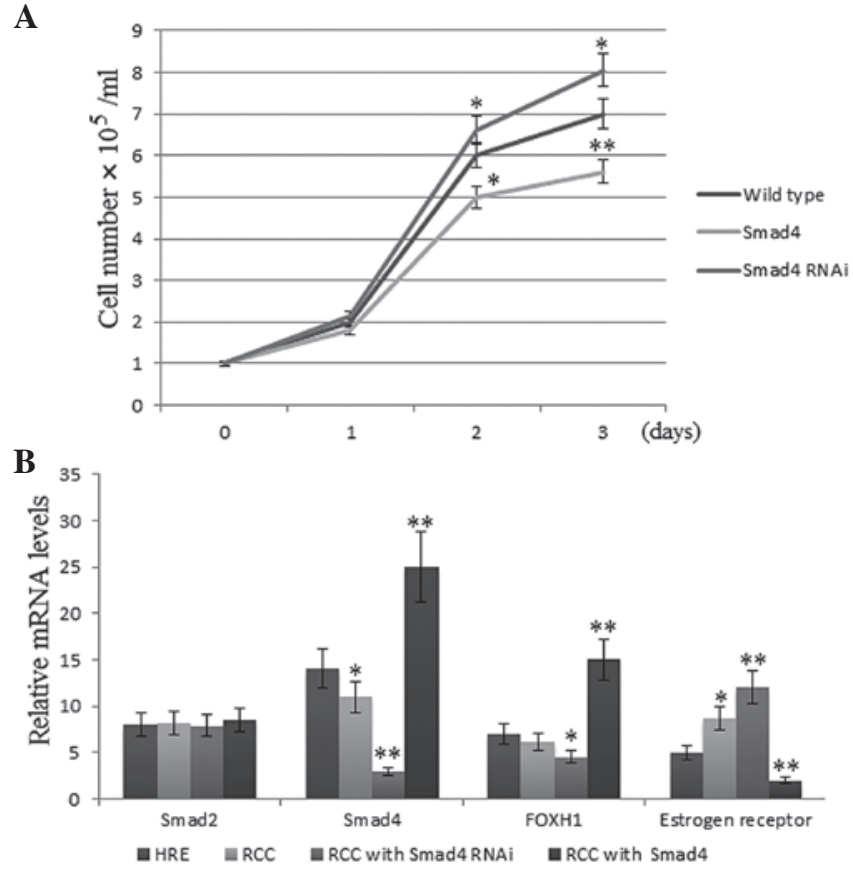

Figure 1. Altered Smad4 expression affects cell RCC cell growth and mRNA expression of Smad2, FOXH1 and estrogen receptor in HRE and RCC cells. (A) Effects of SMad4 gene RNAi and overexpression on the growth rate of RCC. (B) Quantitative polymerase chain reaction of the mRNA and protein levels of Smad2, Smad4, FOXH1 and estrogen receptor in RCC and HRE cells with or without transfection of Smad4 or Smad4 RNAi. The relative mRNA levels were normalized to the signal intensity of GAPDH. Each bar represents the mean \pm the standard deviation of three independent experiments. ${ }^{*} \mathrm{P}<0.05$ and ${ }^{* *} \mathrm{P}<0.01$ vs. the controls. FOXH1, forkhead box protein $\mathrm{H} 1$; RCC, renal cell carcinoma; HRE, normal human renal cells; RNAi, RNA interference.

at $4^{\circ} \mathrm{C}$, and then Protein A/G-plus Agarose was added. The immunoprecipitates were washed three times with lysis buffer and analyzed by western blotting.

Fluorescence microscopy. At $24 \mathrm{~h}$ post-transfection, the cells were fixed with $2 \%$ paraformaldehyde for $10 \mathrm{~min}$, rinsed with phosphate-buffered saline (PBS), and permeabilized with $1 \%$ Triton X-100 for $10 \mathrm{~min}$. The cells were then rinsed with PBS and incubated with monoclonal antibody for $1 \mathrm{~h}$, followed by incubation with secondary antibody for $1 \mathrm{~h}$. The nuclei of the cells were stained with $0.1 \mathrm{~g} / \mathrm{ml}$ DAPI and the cells were observed under a fluorescence microscope (Eclipse E600; Nikon Corp. Toyko, Japan).

Statistical analysis. Values are presented as the mean \pm standard deviation. Statistical analyses were performed using SPSS 20.0 software (International Business Machines, Armonk, NY, USA) and variables were compared using the Students $t$-test. $\mathrm{P}<0.05$ was considered to indicate a statistically significant difference between values.

\section{Results}

Smad4 decreases the growth rate of RCC cells. The growth rate of the OS-RC-2 RCC cells transfected with Smad4 was reduced by $20 \%$ compared with that of the non-transfected cell lines $(\mathrm{P}<0.01)($ Fig. 1A). Conversely, in the RCC cells 
Table I. Characteristics of the patients with renal cell carcinoma and the healthy controls.

\begin{tabular}{llccccc}
\hline & & Healthy & Stage I & Stage II & Stage III & Stage IV \\
\hline Male & Cases (n) & 48 & 29 & 27 & 20 & 13 \\
& Age (years) & $47.5 \pm 6.8$ & $57.3 \pm 5.5$ & $59.4 \pm 9.3$ & $57.1 \pm 8.0$ & $62.3 \pm 9.3$ \\
& BMI (kg/m ${ }^{2}$ ) & $24.7 \pm 5.1$ & $25.5 \pm 8.1$ & $26.3 \pm 6.6$ & $26.9 \pm 6.1$ & $27.7 \pm 7.7$ \\
& Daily calorie intake (kcal) & $2248 \pm 366$ & $2298 \pm 388$ & $2314 \pm 319$ & $2345 \pm 221$ & $2495 \pm 332$ \\
& Sedentary time (h/day) & $6.5 \pm 4.7$ & $7.6 \pm 5.2$ & $8.1 \pm 5.7$ & $9.0 \pm 6.1$ & $9.3 \pm 6.6$ \\
Female & Cases (n) & 32 & 25 & 18 & 12 \\
& Age (years) & $57.9 \pm 7.5$ & $59.8 \pm 6.3$ & $60.7 \pm 7.6$ & $65.1 \pm 8.3$ & $70.2 \pm 8.0$ \\
& BMI (kg/m ${ }^{2}$ ) & $24.9 \pm 5.8$ & $26.9 \pm 7.8$ & $27.8 \pm 9.8$ & $28.8 \pm 9.9$ & $29.9 \pm 10.1$ \\
& Daily calorie intake (kcal) & $1975 \pm 283$ & $1992 \pm 297$ & $2012 \pm 321$ & $2032 \pm 351$ & $2149 \pm 397$ \\
& Sedentary time (h/day) & $6.9 \pm 5.3$ & $8.1 \pm 5.8$ & $8.7 \pm 6.1$ & $9.5 \pm 5.1$ \\
\hline
\end{tabular}

Values are presented as the mean \pm standard deviation. BMI, body mass index (mass $/$ height $^{2}$ ).
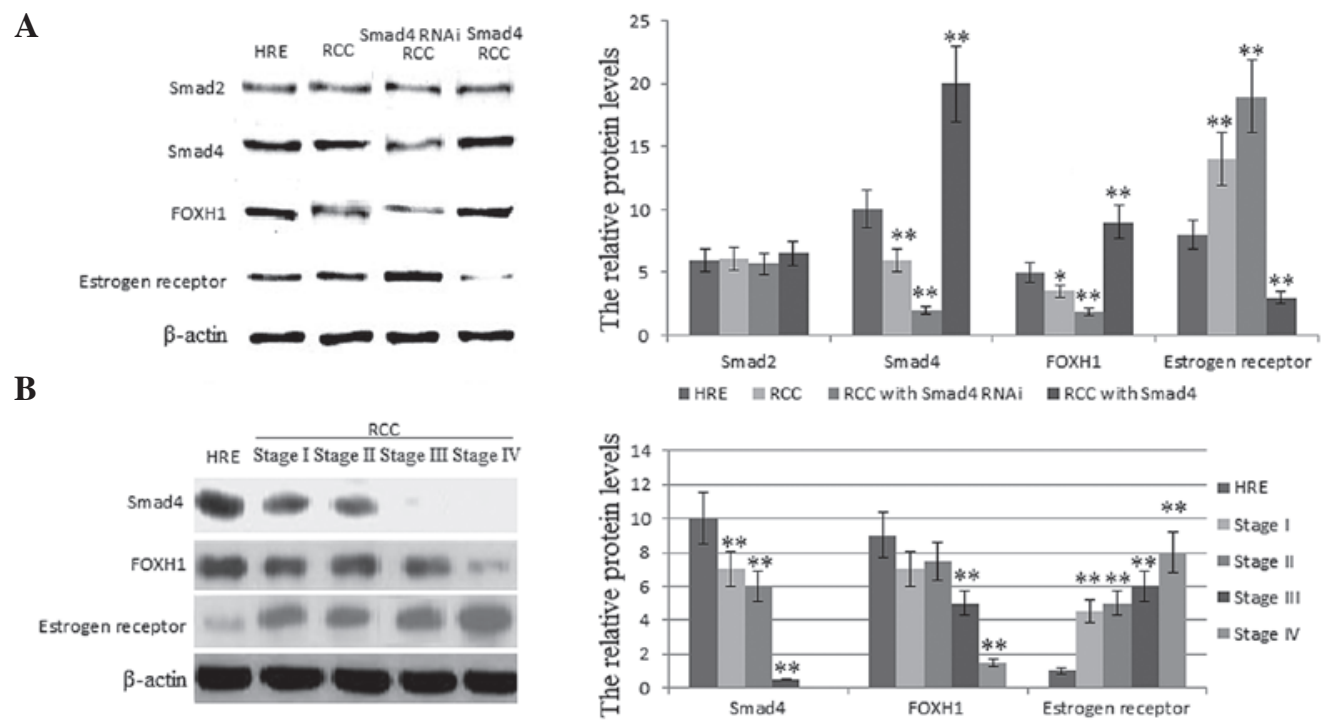

Figure 2. Western blot analysis of the relative protein levels of Smad2, Smad4, FOXH1 and estrogen receptor in renal cells and tissues. (A) Relative protein levels of Smad2, Smad4, FOXH1 and estrogen receptor in OS-RC-2 renal cancer cells and HRE cells. (B) Relative protein levels of Smad4, FOXH1 and estrogen receptor in renal tissues. Relative protein levels were normalized to the signal intensity of $\beta$-actin. Each bar represents the mean \pm the standard deviation of three independent experiments. " $\mathrm{P}<0.05$ and ${ }^{* *} \mathrm{P}<0.01$ vs. the controls. FOXH1, forkhead box protein H1; RCC, renal cell carcinoma; HRE, normal human renal epithelial cells.

transfected with siRNA targeting Smad4, the growth rate was increased by $15 \%$ compared with that of the corresponding non-transfected cell lines $(\mathrm{P}<0.05)$ (Fig. 1A). These results indicated that Smad4 inhibits the proliferation of RCC.

Smad4 expression increases FOXH1 depresses estrogen receptor $m R N A$ levels in RCC cells. Compared with HRE cells, in OS-RC-2 human RCC cells, the mRNA expression levels of Smad4 were reduced, while the expression levels of estrogen receptor were enhanced $(\mathrm{P}<0.01)$ (Fig. 1B). Compared with those of the controls, mRNA levels of Smad4 and FOXH1 were also reduced in RCC cells; however, the difference was not statistically significant. When Smad4 was silenced in RCC cells, the mRNA expression levels of of FOXH1 were slightly reduced and the expression levels of estrogen receptor were markedly increased $(\mathrm{P}<0.01)$. Conversely, when Smad4 was highly expressed in RCC, the mRNA expression levels of
FOXH1 were markedly increased and the expression levels of estrogen receptor were markedly reduced $(\mathrm{P}<0.01)$ (Fig. 1B). Smad2 mRNA levels were the same in RCC and HRE cells and were unaffected by $\mathrm{Smad} 4$ levels $(\mathrm{P}>0.05)$.

Smad4 expression increases FOXH1 depresses estrogen receptor protein levels in RCC cells. Compared with HRE cells, in OS-RC-2 human RCC cells, the protein levels of Smad4 and FOXH1 were reduced, while the levels of estrogen receptor were significantly enhanced (Fig. 2). The protein levels of phospho-Smad2 were stable, indicating that the levels of Smad4 did not affect the degree of phosphorylation of Smad2. When Smad4 was silenced, the protein levels of Smad2 did not change and the protein levels of FOXH1 were significantly reduced, while estrogen receptor levels were markedly increased compared with those of the HRE cells $(\mathrm{P}<0.01)$. Conversely, when Smad4 was highly expressed in RCC, the 
A

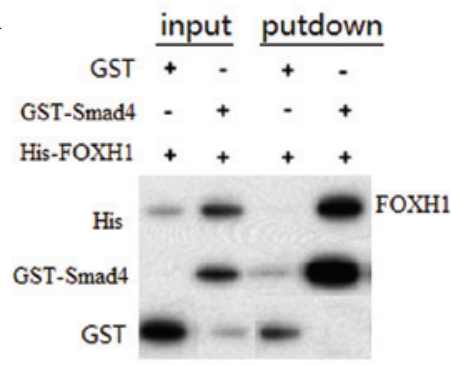

B

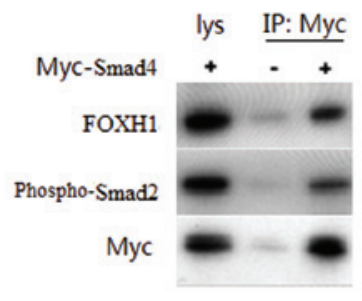

C
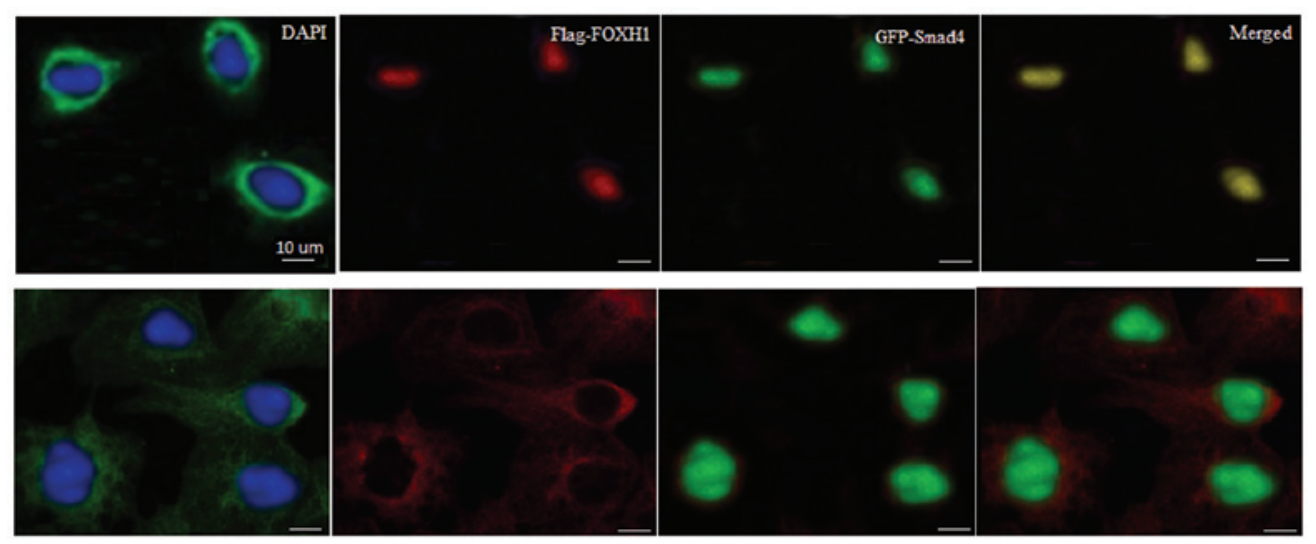

Figure 3. Smad4 interacts with FOXH1. (A) Direct interaction between Smad4 and FOXH1 was revealed by GST pull-down assays. Input and pull-down samples were both subjected to immunoblotting with anti-GST and anti-His antibodies. Input represents $10 \%$ of that used for pull-down. (B) Co-immunoprecipitation of Smad4 and FOXH1 or phospho-FOXH1 in primary neuronal cells. Cell lysates were immunoprecipitated with anti-Myc antibody and analyzed by immunoblotting. (C) Smad4 and FOXH1 colocalize in the nucleus of primary neuronal cells (magnification, x200). Nuclei were stained with DAPI. Top row, fluorescence staining in renal cell carcinoma cells; bottom row, fluorescence staining in normal renal cells. FOXH1, forkhead box protein H1; GST, glutathione S-transferase.

protein levels of FOXH1 were significantly increased and levels of estrogen receptor were markedly reduced compared with those in the HRE cells $(\mathrm{P}<0.01)$. These results suggested that Smad4 is necessary for the repression of the protein expression of estrogen receptor.

Risk of RCC is associated with gender, age, BMI and sedentary lifestyle. A total of 142 subjects $(\mathrm{n}=40$ for normal renal tissues, 18 females/22 males; $n=32$ for stage I RCC, 14 females/18 males; $\mathrm{n}=28$ for stage II RCC, 12 females/16 males; $\mathrm{n}=24$ for stage III RCC, 10 females/14 males; and $n=18$ for stage IV RCC, 7 females/11 males) (Table I) were recruited. When considering the potential confounding factors for increasing the risk of RCC, gender and age were the notable contributing factors (Table I) $(\mathrm{P}<0.05)$. Older participants and male participants were more likey to have a higher risk of RCC. The present study found associations between the BMI and sedentary lifestyles or daily food intake. The BMI increased with a more sedentary lifestyle $(\mathrm{P}<0.01)$ and was additionally associated with the total daily calorie intake. A sedentary lifestyle and high daily food intake were incrementally detrimental to the BMI, which in turn was associated with the development of RCC.

The relative protein levels of phospho-Smad2, Smad4, FOXH1 and estrogen receptor in RCC and HRE tissues. Following the investigation of RCC patient characteristics, the relative protein levels of phospho-Smad2, Smad4, FOXH1 and estrogen receptor were assessed in RCC and HRE tissues. Compared with HRE tissues, in RCC tissues, the protein levels of Smad4 and FOXH1 were reduced, while the levels of estrogen receptor were enhanced (Fig. 2B). The protein expression levels of FOXH1 were significantly reduced, while the expression levels of estrogen receptor were markedly increased $(\mathrm{P}<0.01)$ with increasing $\mathrm{RCC}$ stage.

Smad4 interacts with FOXH1. To confirm the interaction between Smad4 and FOXH1, in vitro GST pull-down assays were performed with recombinant Smad4 and FOXH1. A specific interaction of Smad4 with FOXH1 was observed, but not with GST alone (Fig. 3A). To assess whether Smad4 interacts with FOXH1, a co-immunoprecipitation (Co-IP) assay was performed in primary neuronal cells, and the results revealed an association between Smad4 and Myc-FOXH1 (Fig. 3B). The interaction between Smad4 and FOXH1 in cultured cells indicated that these two types of protein may localize in the same subcellular compartment. To assess the subcellular localization of Smad4 and FOXH1, RCC cells were cotransfected with GFP-Smad4 and Flag-FOXH1. When coexpressed, Smad4 and FOXH1 were colocalized in the nuclei of RCC cells (Fig. 2C, top panel). In normal renal cells, Smad4 was primarily located in cytoplasm, while FOXH1 was localized in the nuclei.

Smad4 forms a complex with phospho-Smad2 and enters the nucleus. This complex interacts with FOXH1 and downregulates the transcriptional activity of estrogen receptor. Therefore, the downregulation of estrogen receptor is likely to inhibit the progression of RCC, as estrogen has been reported to induce renal carcinogenesis in Syrian hamsters (18).

\section{Discussion}

Members of the Smad family are able to activate the transcription of downstream genes by transducing extracellular 
signals from TGF- $\beta$ to the nucleus. There are eight vertebrate Smads, Smad1-8 (13), all of which are classified into three major categories: Receptor-regulated Smads (R-Smads), which include Smad1-3, -5 and -8/9 (20); Smad4, the only common-mediator Smad (co-Smad), which interacts with R-Smads to affect signaling (21); and Smad6 and -7, the inhibitory Smads (I-Smad), which repress the activities of R-Smads and co-Smads (22). Thus, as Smad4 is a separate type of Smad, its function is different from that of the other Smads. Smad4 interacts with phospho-Smad2 or -3 to form an R-Smad/Smad4 complex (23). Although Smad4 has been reported to interact with phospho-Smad2 (24), the present study found that the phosphorylation of Smad2 was not affected by the protein levels of Smad4.

FOXH1 is a transcription factor that mediates signaling by TGF- $\beta$, activin and nodal (24). The biological roles of FOXH1 are diverse. The nodal-FOXH1 signaling pathway has a central role in the anterior-posterior patterning and node formation in mice (25). In addition, FOXH1 has been identified as an androgen receptor repressor (26). FOXH1 does not contain a transcriptional activation domain and requires Smad interaction for transcriptional regulation (16). The formation of the CFSS is necessary for the repression of the transcriptional activities of estrogen receptors.

A number of potential treatments have been explored for inhibiting the progression of RCC. For example, sorafenib has been shown to reduce the risk of RCC-associated mortality; however, the benefit was not statistically significant according to the O'Brien-Fleming threshold (27). Furthermore, sorafenib has a number of adverse side effects, which commonly include diarrhea, rashes, fatigue and hand-foot skin reactions. Hypertension and cardiac ischemia are rare serious side-effects that were more common in patients receiving sorafenib (27). Hence, it is necessary to search for novel therapies with few adverse side-effects. Smad4 is present in the human body and has few side-effects. Thus, Smad4 may be developed as a potential drug for the treatment of RCC. In addition, the present study found that a high daily food intake and sedentary lifestyles were factors that contributed to an increased risk of RCC. Thus, daily physical exercise and a low calorie intake are also important to reduce the risk of RCC.

\section{References}

1. Sheehan JP, Sun MH, Kondziolka D, Flickinger J and Lunsford LD: Radiosurgery in patients with renal cell carcinoma metastasis to the brain: long-term outcomes and prognostic factors influencing survival and local tumor control. J Neurosurg 98: 342-349, 2003.

2. Ljungberg B, Hanbury DC, Kuczyk MA, et al; European Association of Urology Guideline Group for renal cell carcinoma: Renal cell carcinoma guideline. Eur Urol 51: 1502-1510, 2007.

3. Linehan WM: The genetic basis of kidney cancer: implications for management and use of targeted therapeutic approaches. Eur Urol 61: 896-898, 2012.

4. Jacobs JF, Nierkens S, Figdor CG, de Vries IJM and Adema GJ: Regulatory $\mathrm{T}$ cells in melanoma: the final hurdle towards effective immunotherapy? Lancet Oncol 13: e32-e42, 2012.

5. de Jesus-Gonzalez N, Robinson E, Moslehi J and Humphreys BD Management of antiangiogenic therapy-induced hypertension. Hypertension 60: 607-615, 2012.
6. Zhu Y, Xu L, Zhang J, et al: Klotho suppresses tumor progression via inhibiting PI3K/Akt/GSK3 $3 /$ Snail signaling in renal cell carcinoma. Cancer Sci 104: 663-671, 2013.

7. Kim BG, Li C, Qiao W, et al: Smad4 signalling in T cells is required for suppression of gastrointestinal cancer. Nature 441: 1015-1019, 2006

8. Wang LH, Kim SH, Lee JH, et al: Inactivation of SMAD4 tumor suppressor gene during gastric carcinoma progression. Clin Cancer Res 13: 102-110, 2007.

9. Müller N, Reinacher-Schick A, Baldus S, et al: Smad4 induces the tumor suppressor E-cadherin and P-cadherin in colon carcinoma cells. Oncogene 21: 6049-6058, 2002.

10. Losi L, Bouzourene H and Benhattar J: Loss of Smad4 expression predicts liver metastasis in human colorectal cancer. Oncol Rep 17: 1095-1099, 2007.

11. Alazzouzi H, Alhopuro P, Salovaara R, et al: SMAD4 as a prognostic marker in colorectal cancer. Clin Cancer Res 11: 2606-2611, 2005

12. Goto Y, Manabe N, Uchio-Yamada K, et al: Augmented cytoplasmic Smad4 induces acceleration of TGF-beta1 signaling in renal tubulointerstitial cells of hereditary nephrotic ICGN mice with chronic renal fibrosis; possible role for myofibroblastic differentiation. Cell Tissue Res 315: 209-221, 2004.

13. Derynck R and Zhang YE: Smad-dependent and Smad-independent pathways in TGF-beta family signalling. Nature 425: 577-584, 2003.

14. ten Dijke P and Hill CS: New insights into TGF- $\beta$-Smad signalling. Trends Biochem Sci 29: 265-273, 2004.

15. Hoodless PA, Tsukazaki T, Nishimatsu S, Attisano L, Wrana JL and Thomsen GH: Dominant-negative Smad2 mutants inhibit activin/Vg1 signaling and disrupt axis formation in Xenopus. Dev Biol 207: 364-379, 1999.

16. AttisanoL and Wrana JL: Smads as transcriptional co-modulators. Curr Opin Cell Biol 12: 235-243, 2000.

17. Yum J, Jeong HM, Kim S, et al: PKA-mediated stabilization of FoxH1 negatively regulates ERalpha activity. Mol Cells 28: 67-71, 2009.

18. Bhat HK, Hacker HJ, Bannasch P, Thompson EA and Liehr JG: Localization of estrogen receptors in interstitial cells of hamster kidney and in estradiol-induced renal tumors as evidence of the mesenchymal origin of this neoplasm. Cancer Res 53 5447-5451, 1993.

19. Tsui K-H, Shvarts O, Smith Rb, Figlin Ra, deKernion J And Belldegrun A: Prognostic indicators for renal cell carcinoma: a multivariate analysis of 643 patients using the revised 1997 TNM staging criteria. J Urol 163: 1090-1095, 2000.

20. Wu J-W, Hu M, Chai J, et al: Crystal structure of a phosphorylated Smad2: Recognition of phosphoserine by the MH2 domain and insights on Smad function in TGF-beta signaling. Mol Cell 8: 1277-1289, 2001.

21. Shi Y, Hata A, Lo RS, Massagué J and Pavletich NP: A structural basis for mutational inactivation of the tumour suppressor Smad4. Nature 388: 87-93, 1997.

22. Itoh F, Asao H, Sugamura K, Heldin C-H, ten Dijke P and Itoh S: Promoting bone morphogenetic protein signaling through negative regulation of inhibitory Smads. EMBO J 20: 4132-4142, 2001.

23. Ju W, Ogawa A, Heyer J, et al: Deletion of Smad2 in mouse liver reveals novel functions in hepatocyte growth and differentiation. Mol Cell Biol 26: 654-667, 2006.

24. Yao Z, Fenoglio S, Gao DC, et al: TGF-beta IL-6 axis mediates selective and adaptive mechanisms of resistance to molecular targeted therapy in lung cancer. Proc Natl Acad Sci USA 107: 15535-15540, 2010.

25. Yamamoto M, Meno C, Sakai Y, et al: The transcription factor FoxH1 (FAST) mediates Nodal signaling during anterior-posterior patterning and node formation in the mouse. Genes Dev 15: 1242-1256, 2001.

26. Chen G, Nomura M, Morinaga $\mathrm{H}$, et al: Modulation of androgen receptor transactivation by FoxH1 a newly identified androgen receptor corepressor. J Biol Chem 280: 36355-36363, 2005.

27. Escudier B, Eisen T, Stadler WM, et al: Sorafenib in advanced clear-cell renal-cell carcinoma. N Engl J Med 356: 125-134, 2007. 